\title{
APORTES DEL ESTUDIO DE LOS PROCESOS DE FORMACIÓN DE SITIO PARA EL ANÁLISIS DE LOS RESTOS HUMANOS DEL SITIO LAGUNA MUSCAR 2 (LAMADRID, BUENOS AIRES).
}

\section{CONTRIBUTIONS OF THE SITE FORMATION PROCESS STUDIES TO THE HUMAN REMAINS ANALYSIS OF THE SITE LAGUNA MUSCAR 2 (LAMADRID, BUENOS AIRES).}

Cristian A. Kaufmann¹, Jonathan Bellinzoni², María C. Álvarez³, Mariela E. González, Cristian M. Favier-Dubois ${ }^{5}$ y María. P. Barros 6

${ }^{1}$ Instituto de Investigaciones Arqueológicas y Paleontológicas del Cuaternario Pampeano (INCUAPA-CONICET), Facultad de Ciencias Sociales, Universidad Nacional del Centro de la Provincia de Buenos Aires, Olavarría, Argentina.

ckaufman@soc.unicen.edu.ar

2 Departamento de Arqueología, Facultad de Ciencias, Universidad Nacional del Centro de la Provincia de Buenos Aires. jonabellinzoni@gmail.com

3 Instituto de Investigaciones Arqueológicas y Paleontológicas del Cuaternario Pampeano (INCUAPA-CONICET), Facultad de Ciencias Sociales, Universidad Nacional del Centro de la Provincia de Buenos Aires, Olavarría, Argentina. malvarez@soc.unicen.edu.ar

4 Instituto de Investigaciones Arqueológicas y Paleontológicas del Cuaternario Pampeano (INCUAPA-CONICET), Facultad de Ciencias Sociales, Universidad Nacional del Centro de la Provincia de Buenos Aires, Olavarría, Argentina. mgonzalez@soc.unicen.edu.ar

${ }^{5}$ Instituto de Investigaciones Arqueológicas y Paleontológicas del Cuaternario Pampeano (INCUAPA-CONICET), Facultad de Ciencias Sociales, Universidad Nacional del Centro de la Provincia de Buenos Aires, Olavarría, Argentina. cfavier3@gmail.com

${ }^{6}$ Instituto de Investigaciones Arqueológicas y Paleontológicas del Cuaternario Pampeano (INCUAPA-CONICET), Facultad de Ciencias Sociales, Universidad Nacional del Centro de la Provincia de Buenos Aires, Olavarría, Argentina. pbarros@soc.unicen.edu.ar 


\section{Resumen}

En este trabajo se presentan los resultados del análisis de un entierro humano altamente perturbado, recuperado en el sitio Laguna Muscar 2 (partido de Gral. Lamadrid, provincia de Buenos Aires). Los principales objetivos fueron caracterizar el entierro, determinar la modalidad del mismo y evaluar los procesos de formación responsables de su configuración final. Para esto se llevó a cabo la identificación y cuantificación de las unidades anatómicas, en conjunto con el estudio de las modificaciones tafonómicas y la distribución espacial de los elementos en la matriz sedimentaria. Los resultados indicaron que el entierro estaba conformado por un individuo femenino adulto, con deformación craneana artificial de tipo tabular erecta planofrontal y presencia de algunas patologías degenerativas. La modalidad de entierro fue determinada como primaria y el fechado radiocarbónico efectuado arrojó una edad de ca. 2500 años $C^{14}$ AP. Los resultados mostraron la importancia de implementar el estudio de los procesos de formación en el análisis de conjuntos perturbados por animales de hábitos fosoriales. Finalmente, estos datos fueron discutidos junto con los estudios disponibles para la microrregión de la cuenca del arroyo Salado.

Palabras claves: entierro humano, bioturbación, cazadores-recolectores, región pampeana

\section{Abstract}

In this article we present the results of the analysis of a significantly disturbed human burial, recovered at the archaeological site Laguna Muscar 2 (Lamadrid County, province of Buenos Aires). The main objectives were to characterize the burial, to determine the burial type, and to evaluate the site formation processes that were responsible for its final configuration. To achieve these goals, all the anatomical units were identified and quantified, taphonomic modifications were registered, and the spatial distribution of the elements in the sedimentary matrix was studied. The results indicated that the burial was composed by an adult female individual, with artificial (planofrontal) cranial deformation and some degenerative pathology. The type of burial was determined as primary and the radiocarbon date indicated an age of ca. 2500 years $C^{14} A P$. The results showed the importance of implementing the study of formation processes when analyzing assemblages disturbed by fossorial animals. Finally, the resulting data were discussed together with the studies available for the microregion of the Salado creek basin.

Keywords: human burial, bioturbation, hunter-gatherers, Pampas region

\section{Introducción}

Las prácticas mortuorias de los cazadores-recolectores de la región pampeana incluyeron distintas modalidades de entierro a lo largo del Holoceno. Entre estas, se ha reconocido la inhumación primaria, secundaria y la disposición. En los entierros primarios, el cuerpo se deposita articulado, conservando sus relaciones anatómicas (Walthall 1999). En el caso de los entierros secundarios, el cuerpo es depositado en un lugar distinto del original, lo cual implica por lo menos dos procesos: la reducción y la inhumación final (Ubelaker 1978). Por último, en las disposiciones se altera la estructura 
anatómica del cuerpo en forma antrópica, en circunstancias muy cercanas a la muerte. Se trata de un reordenamiento de las partes esqueletarias (Berón y Luna 2007).

Los procesos de formación dificultan las inferencias acerca de la modalidad de entierro, ya que distorsionan las relaciones originales entre los distintos elementos de un cuerpo. Su estudio permite entender cómo se formaron los depósitos, así como identificar las alteraciones post-depositacionales que estos atravesaron (Duday 2009; Lyman 1994; Schiffer 1987; Shipman 1981). En el caso de los restos humanos, la manera en la que un cuerpo es enterrado es importante también, dado que incidirá en la preservación y, por tanto, en lo que podamos inferir respecto de las propias prácticas funerarias y de otros temas relacionados con la biología de los individuos allí representados (Borella et al. 2007; Henderson 1987; Stodder 2008). Existen contextos, como el que se presenta en este trabajo, en los cuales los esqueletos han sido afectados por distintos agentes naturales luego del evento original de enterramiento, aspecto que dificulta la comprensión de la modalidad de entierro. En este tipo de casos, el estudio de los procesos de formación constituye un elemento positivo y necesario para la interpretación de los restos materiales (Tani 1995).

El objetivo de este trabajo fue analizar los procesos de formación responsables de la configuración final del enterratorio del sitio Laguna Muscar 2, localizado en la ciudad de Gral. Lamadrid (provincia de Buenos Aires, Argentina). Con este fin, se identificaron y cuantificaron los elementos óseos presentes. Particularmente, se evaluó la incidencia de diversos factores naturales y culturales en la preservación, integridad esqueletaria y conformación espacial de la inhumación. Conocer la historia tafonómica de este entierro aportó información valiosa para evaluar los agentes que afectaron al conjunto arqueológico y su relación estratigráfica con el resto de los materiales presentes en el sitio. De manera complementaria, uno de los objetivos específicos del trabajo fue enmarcar cronológicamente al enterratorio a través de una datación radiocarbónica y determinar aspectos sexo-etarios del individuo. Hasta el momento, la única modalidad de entierro reconocida para el área es la primaria, por lo cual resulta relevante poder inferir este aspecto para el entierro de Laguna Muscar 2. Finalmente, se integraron los datos obtenidos con aquellos disponibles para los restos humanos de la región.

\section{Antecedentes de la Laguna Muscar}

El lugar del entierro presentado aquí fue denominado sitio Laguna Muscar 2 (LM2) y se localiza sobre la margen oeste de una antigua laguna dentro del casco urbano de la ciudad de Gral. Lamadrid (Figura 1). En cercanías a este sitio, a unos 400 metros y sobre la margen sur de esta misma laguna se halla el sitio Laguna Muscar 1 (LM1). En el año 2010, personal de la policía científica de la provincia de Buenos Aires recuperó en LM1 restos óseos y dientes humanos, hallados durante tareas de construcción llevadas a cabo en una vivienda. El estudio de los huesos realizados por Méndez (2011) y Wiggenhauser (2014) 
informó que el conjunto óseo analizado se encuentra compuesto por 84 elementos. Estos corresponden a dos individuos que formarían parte de un mismo evento de entierro. Ambos presentan cráneos con deformación tabular erecta del tipo lambdoide occipital (planolámbdica), la cual fue considerada por los autores como un indicador cronológico relativo del evento de inhumación, el cual se ubicaría en el Holoceno tardío final (Bernal et al. 2008; Berón y Luna 2009; Perez et al. 2009). Posteriormente, se efectuó un fechado radiocarbónico en el Laboratorio NSF-Arizona AMS, sobre un segundo molar inferior derecho del individuo $\mathrm{N}^{\circ} 1$ que brindó una edad de $429 \pm 42$ años AP (AA104104).

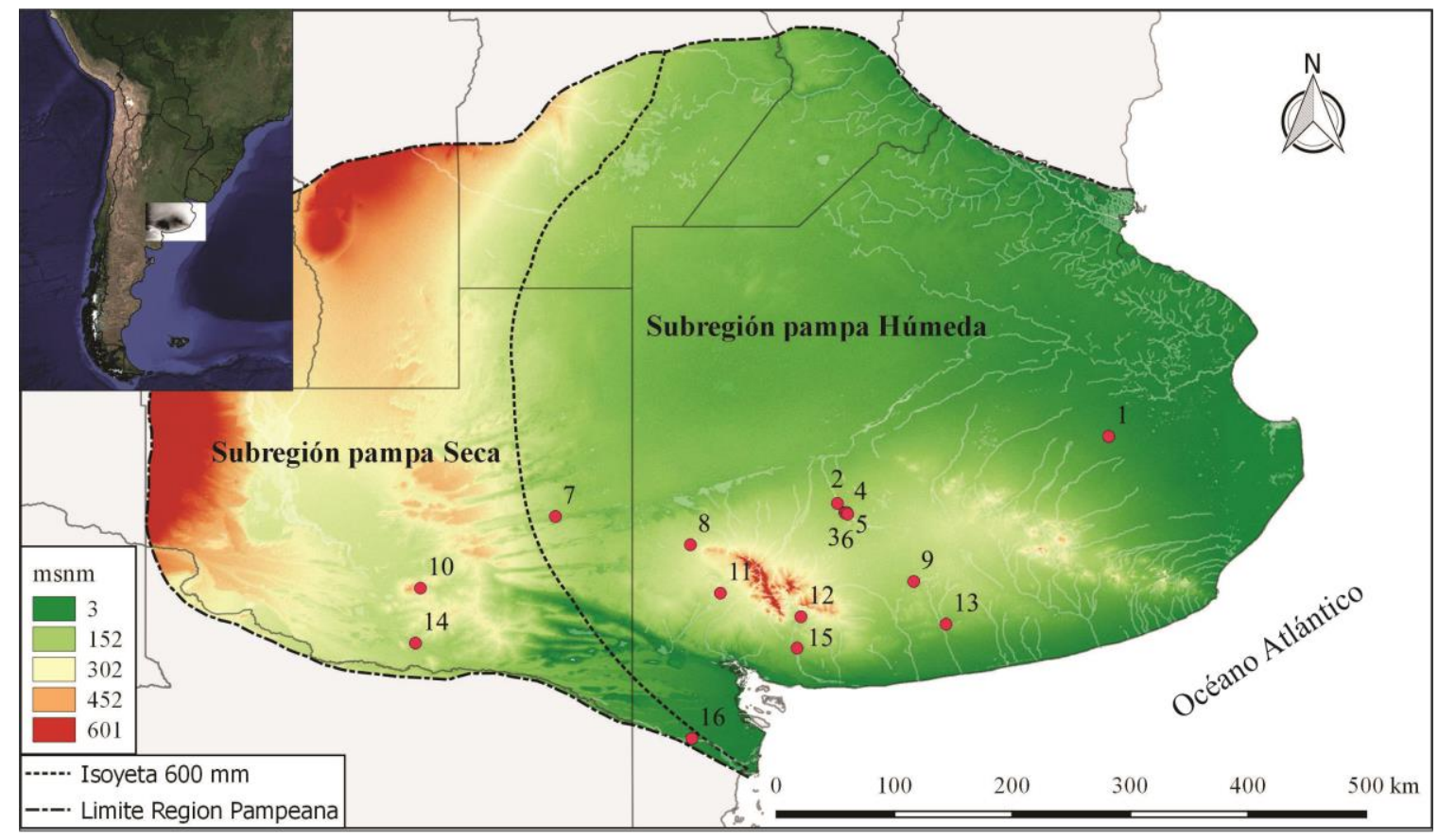

Figura 1. Ubicación de los sitios mencionados en el texto. Referencias: 1=La Colorada, 2=Laguna Seca 2, 3=Laguna Muscar 2, 4=Hangar, 5=Laguna Muscar 1, 6=Lugar de reentierro, 7=Chillué Sitio 3, 8=Laguna de Puán 1, 9=Laguna Tres Reyes, 10=Chenque 1, 11=Laguna Los Chilenos 1, 12=La Toma, 13=Arroyo Seco 2, 14=Tapera Moreira, 15=Paso Mayor 1, 16=La Petrona.

Actualmente la Laguna Muscar se encuentra rellena y en el predio funciona un polideportivo municipal. En septiembre de 2013, tareas de nivelación llevadas a cabo por el municipio expusieron material arqueológico. Ese mismo año se excavaron tres cuadrículas en el lugar de los hallazgos. Estos trabajos permitieron recuperar instrumentos, núcleos y desechos de talla sobre distintas materias primas líticas, cuyos análisis indican actividades ligadas con la producción y uso de instrumentos (Barros et al. 2018). También se registraron especímenes óseos de varios taxones como guanaco (Lama guanicoe), ñandú (Rhea americana), armadillos (Chaetophractus villosus y Dasypus hybridus), vizcacha (Lagostomus maximus), lagarto overo (Tupinambis sp.), una falange humana (Homo sapiens) y numerosos restos de micromamíferos, anuros y reptiles. Uno de los restos de guanaco fue fechado en $4.913 \pm 56$ 
años AP (Barros et al. 2018). Posteriormente, en el año 2015 se ampliaron las excavaciones llegando a cubrir un área de 5,5 $\mathrm{m}^{2}$ y se recuperaron restos óseos y dientes humanos en niveles inferiores del sitio. Algunos de los elementos se hallaban en posición anatómica y varios se encontraban desarticulados. Durante las excavaciones fue posible registrar cuevas de animales cavadores que sugieren la incorporación natural de algunos taxones y el desplazamiento de parte de los restos óseos humanos.

\section{Estratigrafía del sitio}

El análisis geoarqueológico del sitio fue realizado a través del relevamiento de la estratigrafía a escalas puntual (sitio) y local (alrededores). La estratigrafía local se basó en la descripción de un perfil testigo ubicado $100 \mathrm{~m}$ al sur del sitio arqueológico, de unos 60 $\mathrm{cm}$ de profundidad. La estratigrafía a escala puntual consideró dos perfiles expuestos del sitio, los cuales fueron llamados "perfil del sector 1" y "perfil del sector 2", ambos de unos $125 \mathrm{~cm}$ de profundidad.

En la parte superior del perfil testigo (Figura 2a) se desarrolla un horizonte A de suelo (primeros $25 \mathrm{~cm}$ desde la superficie). Por debajo se registra el horizonte AC (entre 25 y 40 $\mathrm{cm}$ desde la superficie) y por último, subyace a éste un horizonte C (a partir de los $45 \mathrm{~cm}$ desde la superficie). Este corresponde a sedimentos eólicos referibles a la Formación La Postrera (Fidalgo et al. 1973), que funcionaron como material parental del suelo. La ausencia de un horizonte B indicaría que no ha tenido mucho tiempo de desarrollo, tratándose de un perfil de suelo joven (Hapludol).

En el sitio, el perfil estratigráfico del sector 1 (cuadrículas 53.1, 54.1 y 55.1) (Figura 2b) presenta características similares al perfil testigo. En este caso, el horizonte A de suelo ubicado en la parte superior del perfil (primeros $10 \mathrm{~cm}$ ) fue parcialmente decapitado por tareas modernas de nivelación, realizadas por el municipio de Gral. Lamadrid en el año 2013. Al igual que en el perfil testigo, por debajo del horizonte $\mathrm{A}$, se observa al horizonte AC (entre 10 y $25 \mathrm{~cm}$ de la superficie decapitada), apoyando sobre el horizonte $C$ (a partir de los $25 \mathrm{~cm}$ de la superficie decapitada) que correspondería a los sedimentos eólicos de La Postrera. En este sector del sitio, el material arqueológico se encuentra circunscripto a los primeros $20 \mathrm{~cm}$ del perfil, e involucra a los horizontes A y AC. De la base del horizonte A proviene el hueso de guanaco sobre el cual se realizó el fechado radiocarbónico que informa una edad referente al Holoceno medio. Por debajo de estos niveles, en el horizonte $\mathrm{C}$ solo fueron halladas microlascas y huesos de pequeños vertebrados de origen natural. Durante las tareas de excavación del sitio, así como en los perfiles de las cuadrículas, se observaron cuevas que interceptaban a los horizontes descriptos. 


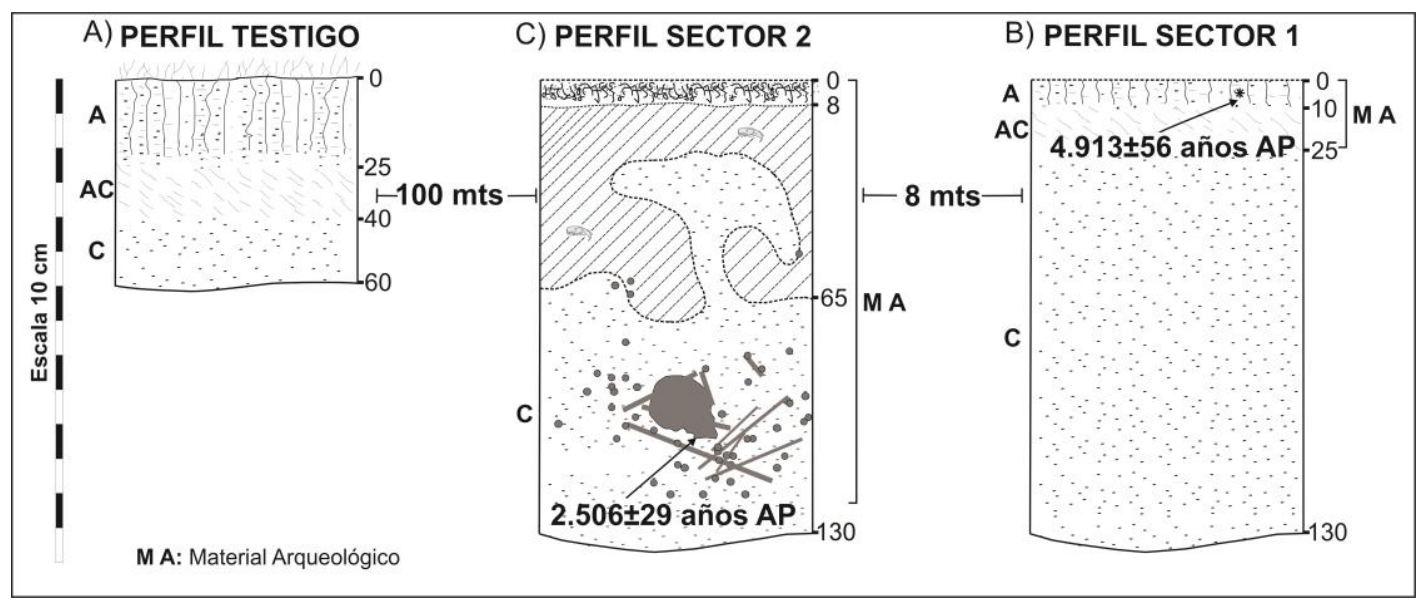

Figura 2. Perfiles estratigráficos relevados en el sitio Laguna Muscar 2.

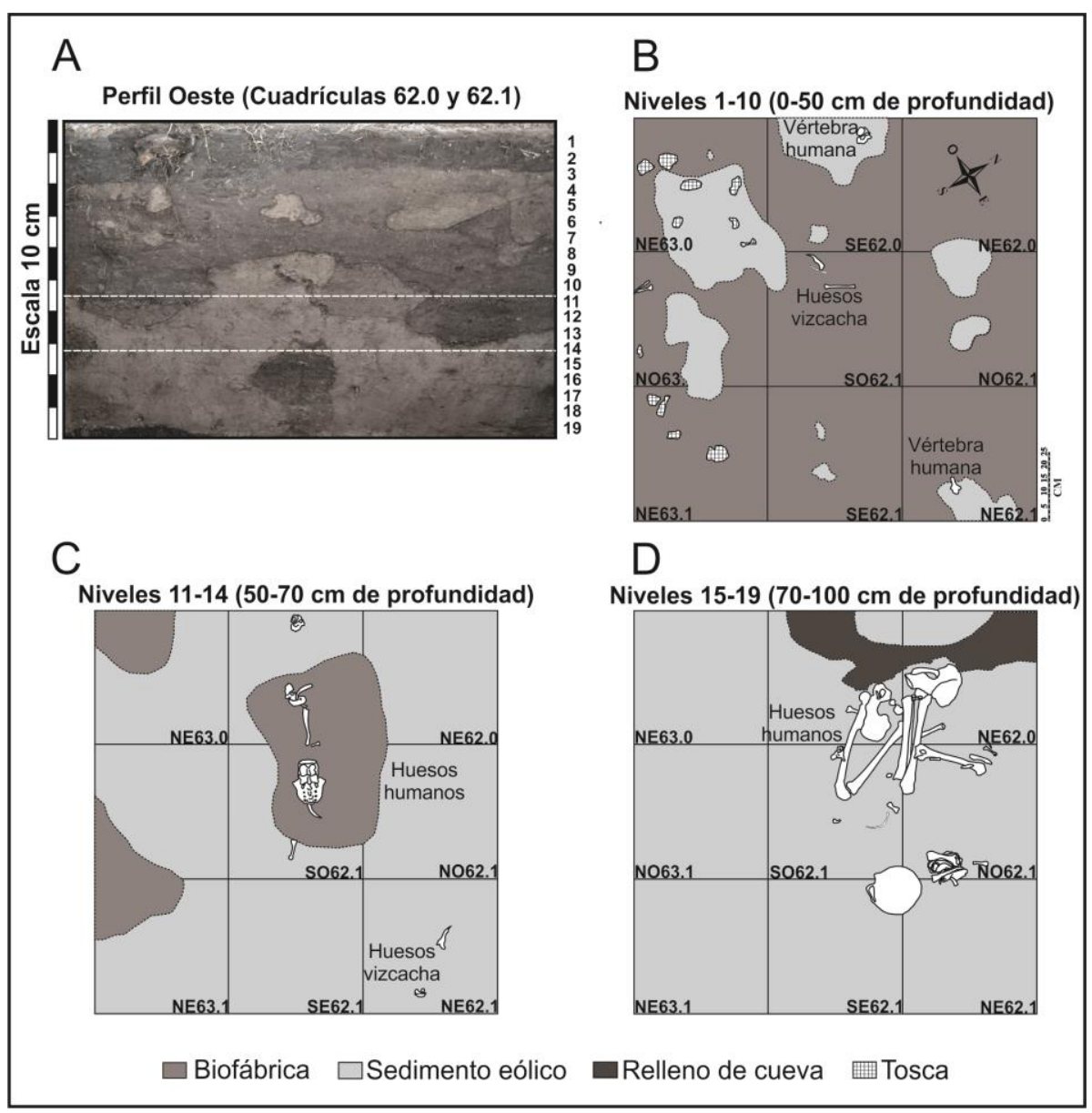

Figura 3. Cuadrículas del sector del enterratorio. A) Perfil estratigráfico de la pared oeste del sitio; B) Planta de excavación de los niveles superiores; C) Planta de excavación de los niveles medios; D) Planta de excavación de los niveles inferiores. 
Por último, el perfil estratigráfico que corresponde al sector 2 (cuadrículas 62.0, 62.1, 63.0 y 63.1) (Figura 2c y 3) presenta importantes modificaciones generadas por animales cavadores, las cuales alteraron de manera significativa las secuencias estratigráficas anteriormente descriptas. Se observa que la porción superior, de unos $8 \mathrm{~cm}$ de espesor, presenta un color y textura que se diferencia de los sedimentos subyacentes. Este estrato contenía una mezcla de materiales de distinta cronología que comprendieron huesos de guanaco, artefactos líticos y fauna doméstica de origen europeo (Bos taurus) asociados en lo que parece constituir un relleno moderno. Por debajo del mismo y hasta los $70 \mathrm{~cm}$ de profundidad se observa un depósito de mezcla por bioactividad (biofábrica) que se corresponde con el relleno de cuevas. Estos sedimentos contenían huesos de animales modernos (e.g. vizcacha, comadreja), especímenes de guanaco y artefactos líticos. Durante la excavación de estos niveles estratigráficos se relevaron numerosas estructuras correspondientes a cuevas de animales fosoriales, acumulaciones de tosca y relictos del sedimento eólico basal. A una profundidad de $50 \mathrm{~cm}$ y asociados con este sedimento eólico fueron registrados los primeros huesos aislados que correspondían al enterratorio humano. Por debajo de los $70 \mathrm{~cm}$, los sedimentos eólicos del horizonte $\mathrm{C}$ poseen mayor integridad, aunque también se relevaron numerosas cuevas. Es en estos niveles y llegando hasta los $95 \mathrm{~cm}$ de profundidad donde se hallaba depositado el enterratorio humano (Figura 3).

\section{Aspectos metodológicos del estudio del entierro}

Se realizaron diversas tareas como: a) identificación de los elementos anatómicos, determinación del grado de fusión y lateralidad, relevamiento de patologías óseas y estimación sexo-etaria (Buikstra y Ubelaker 1994; White y Folkens 2000); b) análisis cuantitativos para precisar el porcentaje de elementos óseos representados, conocer el número total de unidades óseas y de individuo/s representado/s y reconocer el grado de fragmentación de los materiales; c) análisis macroscópicos de los efectos tafonómicos (sensu Lyman 1994) registrados en la superficie cortical; y d) evaluación de la disposición de los elementos óseos para determinar la modalidad inhumatoria e identificar los procesos postdepositacionales vinculados con los desplazamientos horizontales y verticales.

En la primera aproximación analítica se identificaron las distintas unidades óseas y su correspondiente lateralidad. Asimismo, se registraron los rasgos indicativos de los estados de fusión de cada hueso así como los indicadores morfológicos diagnósticos del sexo y la edad probable de muerte (Steele y Bramblett 2005; White y Folkens 2000). Como segundo paso, se calcularon los índices cuantitativos. En este trabajo el NISP fue empleado como una forma de medir la cantidad de fragmentos óseos asignados a un determinado hueso, porción del mismo, o región del esqueleto. El NMI se obtuvo observando la frecuencia correspondiente al elemento anatómico más abundante en la 
muestra, considerando lateralidad, estado de fusión y tamaño. Para el NME se calculó la frecuencia de representación de cada una de las unidades anatómicas que componen el esqueleto, independientemente de su lateralidad. El porcentaje de supervivencia (\%Sup.) se obtuvo comparando porcentualmente las frecuencias halladas para cada parte anatómica con la frecuencia esperada acorde al NMI (Brain 1981; Klein y Cruz Uribe 1984; Lyman 1994).

Posteriormente se realizó el análisis de las modificaciones presentes sobre la superficie cortical de los especímenes a través de la observación macroscópica de los restos, utilizando lupa binocular de 40x. Distintas variables tafonómicas fueron consideradas, entre las que se encuentran: meteorización, marcas de raíces, depositación química (carbonato de calcio y óxido de manganeso), integridad (<25\%, 25-50\%, 51-75\% y 76$100 \%$ ), pérdida ósea (tipo: postdepositacional o reciente; tamaño: pequeña, moderada, extensiva), tipo de fractura (postdepositacional o reciente), fragmentación (tamaños promedio: $1-5 \mathrm{~cm}, 5-10 \mathrm{~cm}$ y $>10 \mathrm{~cm}$ ), actividad de roedores, acción de carnívoros, huellas de corte, deterioro químico (rasgos: líneas de desecación, estrías de desecación, grietas, exfoliación, astillamiento), marcas de excavación y de limpieza y preservación macroscópica (muy buena, buena, regular y mala) (Behrensmeyer 1978; Binford 1981; González 2013, 2014, Gutiérrez 2004; Johnson 1985; Lyman 1994; Lyman y Fox 1989; Villa y Mahieu 1991).

Finalmente, se evaluaron los desplazamientos postdepositacionales que tuvieron los restos óseos del entierro. Este aspecto, junto a las variables tafonómicas antes mencionadas, permite discutir la modalidad del enterratorio, la posición del esqueleto y los procesos postdepostacionales que afectaron al mismo. Como primer paso se examinaron los datos obtenidos en el campo, donde se siguió un protocolo de extracción registrando los especímenes de manera bidemensional y tridimensional a través de fotografías y estación total. El análisis de los mapeos de campo y las fotografías del sitio permitieron registrar los desplazamientos espaciales horizontales y verticales, considerando las distancias entre unidades anatómicas así como su posición en relación con la estructura anatómica original. Las distancias entre los especímenes vinculados fueron calculadas a través del programa QGIS 3.0.2.

Posteriormente, se llevaron a cabo remontajes de tipo mecánico y anatómico (Todd 1987; Tood y Frison 1992; Todd y Stanford 1992). Los remontajes mecánicos se realizaron acoplando dos o más fragmentos pertenecientes a un mismo elemento óseo a partir de la superficie de fractura. Por otra parte, los remontajes anatómicos consistieron en la recomposición de las relaciones intraesqueletarias entre elementos óseos. Las mismas se reconocieron considerando dos variantes: las uniones bilaterales e intermembrales. Las primeras refieren a la correspondencia bilateral entre elementos anatómicos pares (e.g., húmero derecho y húmero izquierdo). Las segundas consisten en las relaciones entre 
elementos que articulan entre sí (considerando todos los tipos de articulaciones presentes en el esqueleto humano). Entre ellas se incluyen las rearticulaciones (Marín 2004: 26). Este tipo de unión pone en relación especímenes óseos que fueron hallados separados pero que forman parte un elemento complejo. Ejemplos de este tipo de remontaje son las uniones entre dientes aislados y alvéolos de mandíbulas/maxilares (articulación fibrosa), unión entre coxal izquierdo y derecho a través de la sínfisis púbica (articulación cartilaginosa secundaria) y uniones entre epífisis no fusionadas y sus correspondientes metáfisis (articulación cartilaginosa primaria).

\section{Resultados}

Características generales y análisis tafonómicos

A través de la excavación del enterratorio se recuperaron 101 especímenes óseos y 11 fragmentos de dientes aislados. Los análisis anatómicos, sexo-etarios y tafonómicos permiten establecer que se trata de un único individuo femenino adulto (edad aproximada entre 35 y 45 años). Este individuo presenta una deformación craneana artificial antero-posterior en la que los huesos frontal y occipital se encuentran aplanados, conocida como tabular erecta planofrontal (Figura 4). Varios elementos esqueletales presentan evidencias de patologías de tipo degenerativo, tales como eburnaciones y osteofitos. Se realizó un fechado radiocarbónico en el Laboratorio NSFArizona AMS, sobre el tercer molar inferior izquierdo que brindó una edad de 2.506 +29 años AP (AA106808).

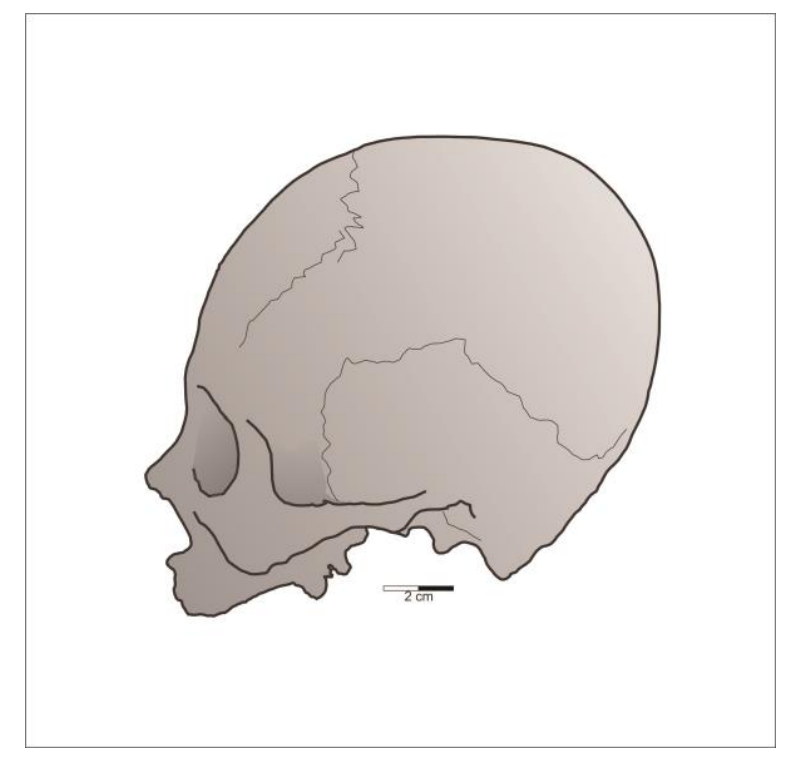

Figura 4. Esquema en base a la fotografía del cráneo del esqueleto 1. Se observa la deformación craneana de tipo tabular erecta planofrontal. 


\begin{tabular}{|c|c|c|c|c|c|c|c|}
\hline & $\begin{array}{l}\text { Unidad } \\
\text { anatómica }\end{array}$ & $\begin{array}{c}\text { NISP } \\
\text { Derecho }\end{array}$ & $\begin{array}{l}\text { NISP } \\
\text { Axial }\end{array}$ & $\begin{array}{c}\text { NISP } \\
\text { Izquierdo }\end{array}$ & NME & PR & PRA \\
\hline \multirow{3}{*}{ Cráneo } & Cráneo & - & 1 & - & 1 & 100 & \multirow{3}{*}{100} \\
\hline & Dientes aislados & - & 11 & - & - & - & \\
\hline & Mandíbula & - & 1 & - & 1 & 100 & \\
\hline \multirow{4}{*}{ Vértebras } & V. cervical & - & 1 & - & 1 & 14,28 & \multirow{4}{*}{41,37} \\
\hline & V. toráxica & - & 9 & - & 7 & 58,33 & \\
\hline & V. lumbar & - & 4 & - & 4 & 80 & \\
\hline & V. coxis & - & 0 & - & 0 & 0 & \\
\hline \multirow{2}{*}{$\begin{array}{l}\text { Cintura } \\
\text { pélvica }\end{array}$} & Sacro & - & 1 & - & 1 & 100 & \multirow{2}{*}{100} \\
\hline & Coxal & 1 & - & 1 & 1 & 100 & \\
\hline \multirow{2}{*}{ Tórax } & Esternón & - & 4 & - & 1 & 100 & \multirow{2}{*}{64} \\
\hline & Costillas & 6 & 6 & 9 & 15 & 62,5 & \\
\hline \multirow{2}{*}{$\begin{array}{l}\text { Cintura } \\
\text { escapular }\end{array}$} & Clavícula & - & 1 & - & 1 & 50 & \multirow{2}{*}{75} \\
\hline & Escápula & 1 & - & 1 & 2 & 100 & \\
\hline \multirow{3}{*}{ Brazos } & Húmero & 1 & - & 1 & 2 & 100 & \multirow{3}{*}{50} \\
\hline & Cúbito & - & - & - & - & 0 & \\
\hline & Radio & 1 & - & - & 1 & 50 & \\
\hline \multirow{6}{*}{ Manos } & Carpo & 3 & 1 & 3 & 7 & 43,75 & \multirow{6}{*}{48,14} \\
\hline & Metacarpo & 2 & 1 & 1 & 4 & 40 & \\
\hline & Falange px. & - & 6 & - & 6 & 60 & \\
\hline & Falange md. & - & 2 & - & 2 & 25 & \\
\hline & Falange ds. & - & 6 & - & 6 & 60 & \\
\hline & Falange indet. & - & 1 & - & 1 & - & \\
\hline \multirow{4}{*}{ Piernas } & Fémur & 1 & - & 1 & 2 & 100 & \multirow{4}{*}{75} \\
\hline & Rótula & - & - & - & - & 0 & \\
\hline & Tibia & 1 & - & 1 & 2 & 100 & \\
\hline & Peroné & 1 & - & 1 & 2 & 100 & \\
\hline \multirow{8}{*}{ Pies } & Astrágalo & 1 & - & 1 & 2 & 100 & \multirow{7}{*}{34,61} \\
\hline & Calcáneo & - & - & 1 & 1 & 50 & \\
\hline & Tarso & 1 & 1 & 1 & 3 & 30 & \\
\hline & Metatarso & 2 & - & 2 & 4 & 40 & \\
\hline & Falange px. & - & 6 & - & 6 & 60 & \\
\hline & Falange md. & - & 1 & - & 1 & 12,5 & \\
\hline & Falange ds. & - & 1 & - & 1 & 10 & \\
\hline & & 22 & 65 & 24 & 99 & 47,6 & \\
\hline
\end{tabular}

Tabla 1. NISP, NME y porcentaje de supervivencia esqueletaria individual (PR) y agrupada (PRA) del esqueleto 1. 
Los resultados del análisis tafonómico muestran que los elementos óseos recuperados presentan, en general, una muy buena preservación. De las variables consideradas, sólo se registraron cinco de ellas y con bajas frecuencias. Las fracturas y la pérdida ósea son las modificaciones que más especímenes afectaron $(35,7 \%$ y 31,2\%, respectivamente). Las marcas de raíces se presentan de manera aislada en el $17 \%$ de los elementos, mientras que las precipitaciones de carbonato y manganeso también exhiben bajas frecuencias $(35,7 \%$ y $20,5 \%$, respectivamente) y se distribuyen irregularmente sobre cada espécimen. Ambas depositaciones químicas se manifiestan con baja intensidad en cuanto a su modo de precipitación; por un lado, el manganeso con pequeñas y escasas dendritas y por otro, el carbonato de calcio en forma de una fina capa pulverulenta. En cuanto a la integridad de los elementos, la mayoría $(82,1 \%)$ se encuentra dentro del rango $76-100 \%$.

Representación esqueletaria

Se determinó un número mínimo de 99 elementos óseos (NME) (Tabla 1). El individuo presenta una supervivencia esqueletaria del $47,6 \%$ y se encuentran representadas, con cierta variabilidad, todas las regiones anatómicas del esqueleto. Los segmentos anatómicos más incompletos corresponden al antebrazo, manos y pies. En la columna vertebral, el segmento menos representado es el cervical. Las vértebras torácicas y costillas poseen una representación media. El cráneo, cintura pélvica, brazos y piernas se encuentran muy bien representados (Tabla 1$)$.

Procesos postdepositacionales, modalidad del entierro y posición del esqueleto

El análisis indica que los segmentos esqueletarios que se hallaban incompletos también son los que presentan mayor grado de dispersión. Los huesos de manos y pies, costillas y vértebras (a excepción de tres lumbares) se encontraban en su totalidad desarticulados. Estos especímenes se hallaron dispersos en el plano horizontal y vertical. En este último caso, el desplazamiento abarcó desde el nivel 9 al 20 de la excavación (ca. $55 \mathrm{~cm}$ ). La determinación anatómica, lateralidad y estado de fragmentación de los especímenes permitió establecer 27 vinculaciones óseas con un promedio de desplazamiento de $42,3 \mathrm{~cm}$. Entre estas, se hallan: una unión mecánica entre dos fragmentos del esternón que se encontraban a $38 \mathrm{~cm}$ y dos uniones bilaterales que comprenden al húmero derecho e izquierdo $(31,1 \mathrm{~cm})$ y al segundo metatarso derecho e izquierdo $(83,8 \mathrm{~cm})$. Asimismo, se realizaron 23 uniones intermembrales que involucraron especímenes óseos del cráneo, miembros superiores, porciones distales de miembros inferiores y pies y cintura pélvica (Tabla 2 y Figura 5). La distancia de desplazamiento de los elementos que integran estas unidades anatómicas fue similar, con un promedio de $47 \mathrm{~cm}$ para el miembro superior derecho, 42,1 para el miembro superior izquierdo, 31,1 para el pie derecho, 46,2 para el pie izquierdo y 53,7 para la 
cintura pélvica (Tabla 2). Se determinaron dos rearticulaciones, una de las cuales vinculó un conjunto de dientes sueltos con la mandíbula y cráneo $(31,7 \mathrm{~cm})$, y la otra, el coxal izquierdo y derecho $(8,4 \mathrm{~cm}$ ) (Tabla 2 y Figura 5). Finalmente, es interesante mencionar que una costilla fue hallada en el perfil de la zanja que intercepta al sitio a unos 2,8 m y una falange proximal del pie se halló en una cuadrícula ubicada a $8 \mathrm{~m}$ del enterratorio. Por el momento resulta difícil establecer si estos huesos son efectivamente parte del mismo individuo. El hallazgo aislado de elementos esqueletales humanos constituye un ejemplo de cómo distintos procesos de formación y agentes tafonómicos intervinieron en el sitio desplazando huesos.

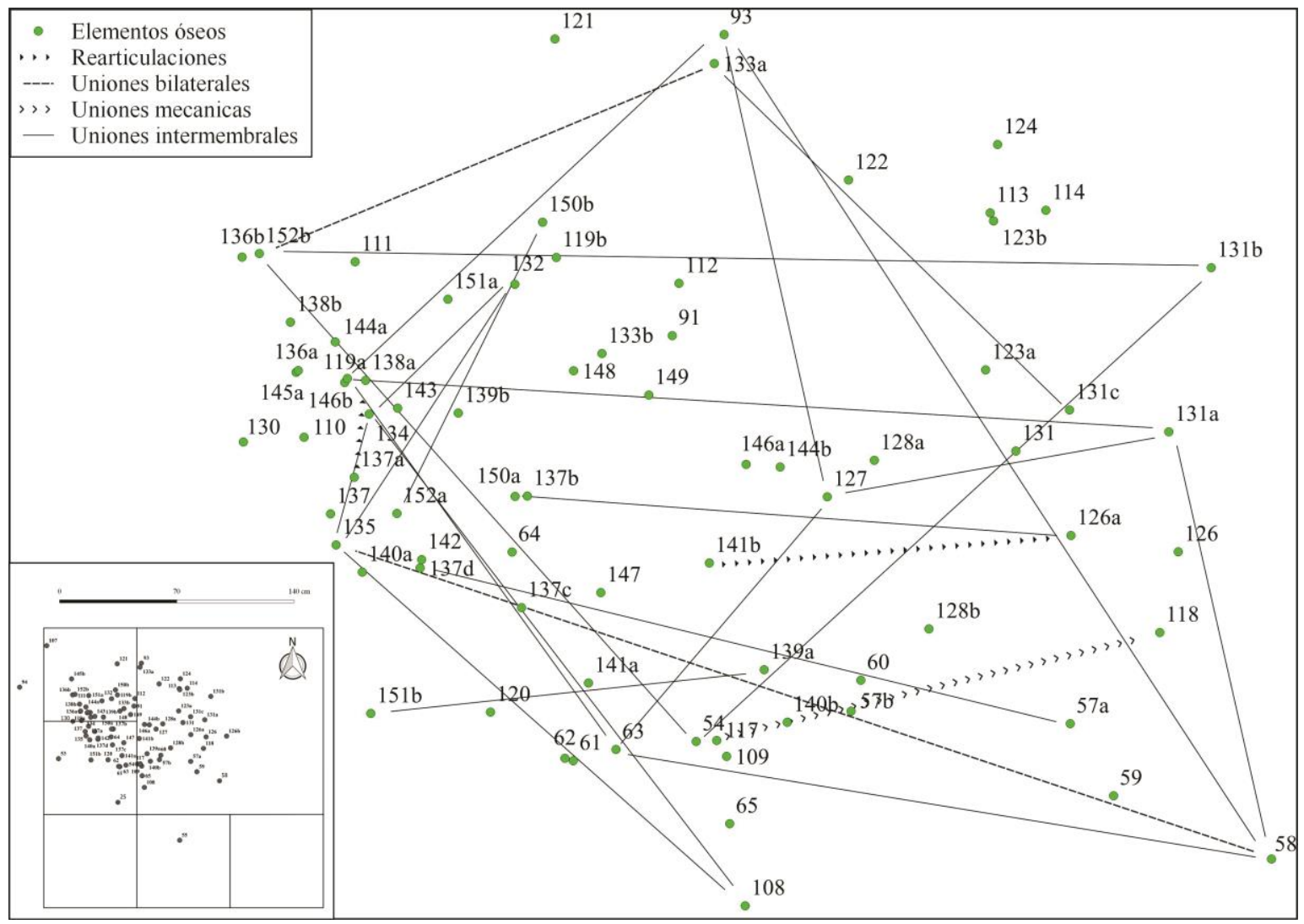

Figura 5. Uniones óseas establecidas en el enterratorio. En la tabla 2 se detallan los tipos de uniones representadas en el gráfico. 


\begin{tabular}{|c|c|c|c|c|c|c|c|}
\hline Unión & Región & ID & Elemento & ID & Elemento & $\mathrm{Cm}$ & Media \\
\hline A & Tórax & 117 & Frag. Esternón & 118 & Frag. esternón & 38,8 & 38,8 \\
\hline \multirow{2}{*}{ B } & $\begin{array}{l}\text { Miembro } \\
\text { superior }\end{array}$ & 152 & Húmero der. & 133 & Húmero izq. & 31,12 & 31,12 \\
\hline & Pies & 135 & $2^{\text {do }}$ Metatarso der & 58 & $\begin{array}{l}\text { 2do metatarso } \\
\text { izq. }\end{array}$ & 83,84 & 83,84 \\
\hline \multirow{22}{*}{$\mathrm{C}$} & \multirow{5}{*}{$\begin{array}{l}\text { Miembro } \\
\text { superior der. }\end{array}$} & 54 & Clavícula & 131 & Escápula px. & 36,7 & \multirow{5}{*}{47} \\
\hline & & 152 & Húmero px. & 131 & Escápula px. & 67,1 & \\
\hline & & 54 & Clavícula & 152 & Húmero px. & 55,3 & \\
\hline & & 150 & Radio ds. & 126B & Trapezoide & 47,8 & \\
\hline & & 152 & Húmero ds. & 150 & Radio px. & 28,1 & \\
\hline & $\begin{array}{l}\text { Miembro } \\
\text { superior izq. }\end{array}$ & 133 & Húmero px. & 131 & Escápula px. & 42,1 & 42,1 \\
\hline & \multirow{5}{*}{ Pie der. } & 134 & Astrágalo & 108 & $3^{\mathrm{er}}$ metatarso & 53,4 & \multirow{5}{*}{31,12} \\
\hline & & 134 & Astrágalo & 135 & $2^{\text {do }}$ metatarso & 12,8 & \\
\hline & & 134 & Astrágalo & 132 & $1^{\mathrm{er}}$ cuneiforme & 16,6 & \\
\hline & & 108 & $3^{\text {er }}$ metatarso & 135 & $2^{\text {do }}$ metatarso & 46,3 & \\
\hline & & 135 & $2^{\text {do }}$ metatarso & 132 & $1^{\text {er }}$ cuneiforme & 26,9 & \\
\hline & \multirow{10}{*}{ Pie izq. } & 146 & Tibia ds. & 63 & Calcáneo & 39 & \multirow{10}{*}{46,21} \\
\hline & & 146 & Tibia ds. & 131 & Astrágalo & 69,8 & \\
\hline & & 146 & Tibia ds. & 93 & Cuboide & 43,5 & \\
\hline & & 139 & Tibia px. & 151 & Peroné px. & 33,9 & \\
\hline & & 63 & Calcáneo & 127 & 1er metatarso & 28 & \\
\hline & & 63 & Calcáneo & 58 & $2^{\text {do }}$ metatarso & 56,7 & \\
\hline & & $131^{\mathrm{a}}$ & Astrágalo & 127 & $1^{\mathrm{er}}$ metatarso & 29,7 & \\
\hline & & 131 & Astrágalo & 58 & $2^{\text {do }}$ metatarso & 37 & \\
\hline & & 93 & Cuboide & 127 & $1^{\mathrm{er}}$ metatarso & 40,3 & \\
\hline & & 93 & Cuboide & 58 & $2^{\text {do }}$ metatarso & 84,2 & \\
\hline & Cintura pélvica & 57 & Sacro & 137 & Coxal derecho & 53,7 & 53,7 \\
\hline \multirow[t]{2}{*}{ D } & Cráneo & 126 & $\begin{array}{l}\text { Cráneo- } \\
\text { mandíbula }\end{array}$ & 0 & Dientes & 31,7 & 31,7 \\
\hline & Cintura pélvica & 137 & S. púbica der. & 138 & S. púbica izq. & 8,4 & 8,4 \\
\hline
\end{tabular}

Tabla 2. Vinculaciones óseas establecidas en el esqueleto 1. A: unión mecánica, B: unión bilateral, C: unión intermebral, D: rearticulaciones 


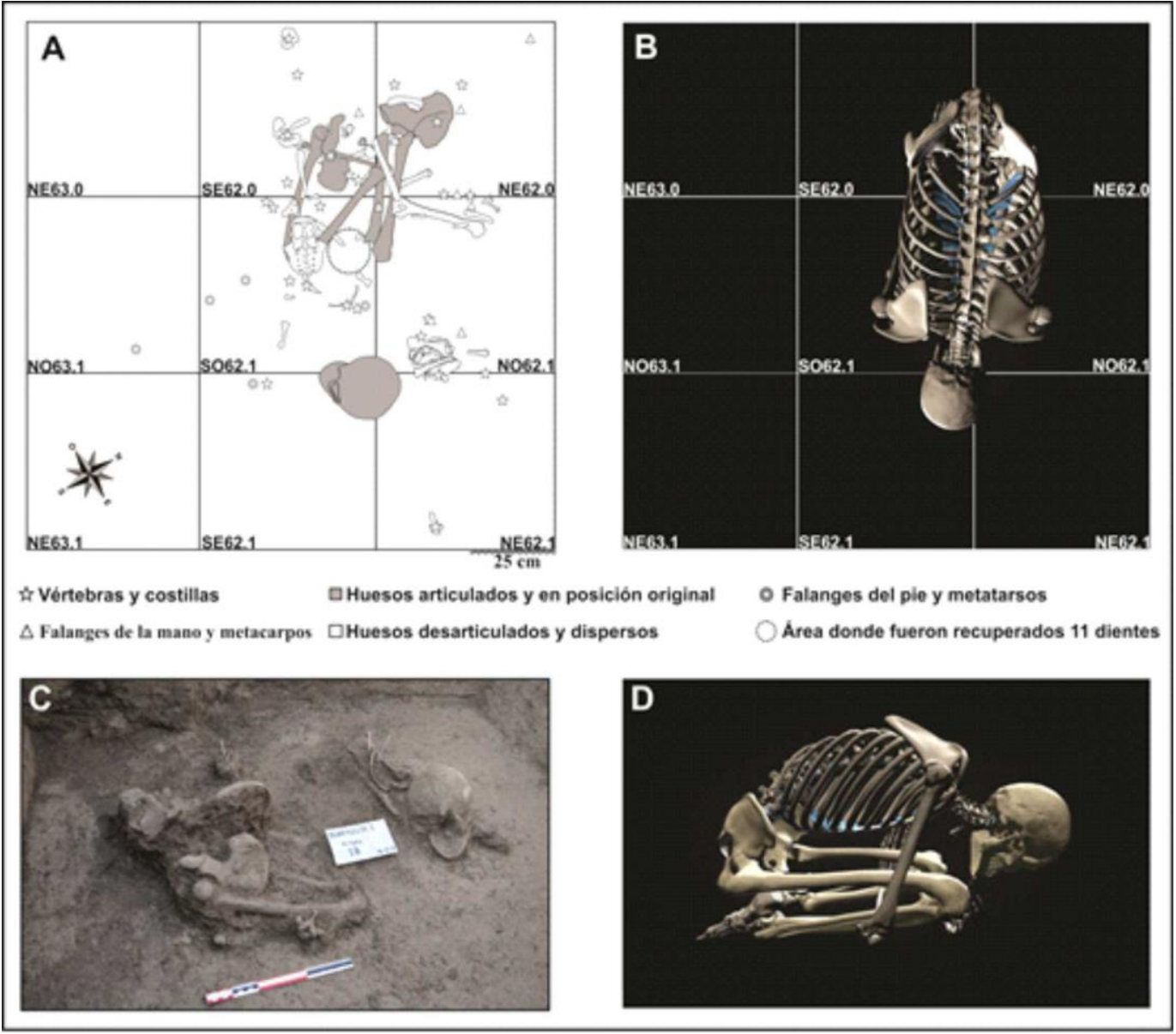

Figura 6. A) Mapeo del individuo 1; B) Vista dorsal de la posición original que tendría el individuo; C) Vista del esqueleto durante la excavación, y D) vista lateral de la posición original que tendría el esqueleto.

Cuando se considera el grado de articulación de los restos y sus posiciones anatómicas se observa que algunos elementos se hallaban articulados y muy cerca de su posición original. Otros se encontraban en segmentos articulados integrados por dos o tres especímenes articulados sin relación anatómica al resto del esqueleto y, finalmente, algunos elementos estaban desarticulados y sin posición anatómica. El primer caso es el que genera información valiosa para inferir la posición original del esqueleto y su relación con la depositación cultural del mismo. El miembro inferior (fémur, tibia y astrágalo) y el coxal derecho se presentan articulados y en posición original. Este se encuentra flexionado, con el coxal y el fémur formando un ángulo cerrado. El miembro inferior izquierdo (fémur, tibia y peroné) también se halla articulado y flexionado. En el caso del coxal, se desplazó algunos centímetros hacia atrás con respecto al coxal derecho, quedando el pubis de ambos separado por 15 centímetros. El cráneo y la mandíbula se encontraban articulados, apoyando sobre la sínfisis mandibular, con una leve rotación hacia la derecha. Estos especímenes, si bien podrían haber experimentado un pequeño desplazamiento 
hacia el este, no se encontrarían muy lejos de su posición original. El conjunto de dientes sueltos, hallados en un sector acotado entre los miembros inferiores, podría indicar la ubicación original del cráneo y la mandíbula. El resto de los huesos ha sufrido desarticulación y desplazamiento horizontal y vertical que provocó la ruptura de las relaciones anatómicas. Entre estos se encuentra un segmento articulado formado por el sacro y tres vértebras lumbares, que sufrió un desplazamiento vertical y quedó situado a unos $25 \mathrm{~cm}$ de los coxales. El resto de los huesos que conforman los brazos, manos, pies, columna vertebral y caja torácica se encuentran desarticulados y dispersos. Los especímenes faltantes de estos segmentos anatómicos posiblemente sufrieron desplazamientos por fuera del área excavada. La estructura espacial de los huesos que conforman el esqueleto sugiere que se trata de un enterratorio primario, en el cual el individuo fue depositado en posición decúbito ventral con los miembros inferiores estrechamente flexionados y una orientación sureste (Figura 6).

\section{DISCUSIÓN Y CONCLUSIONES}

Procesos de formación que afectaron al entierro

El sector de la laguna en el cual se ubica el sitio LM2 fue impactado por actividades antrópicas modernas vinculadas al zanjeo de drenaje hídrico y nivelación de la superficie del terreno y por procesos naturales tiempo transgresivos como es la acción de animales cavadores. Esto se condice con estudios previos en la región pampeana, donde los mamíferos cavadores constituyen agentes tafonómicos recurrentes en la perturbación de los sitios arqueológicos y, en particular, de yacimientos con entierros humanos (Bayón et al. 2004; Frontini y Deschamps 2007; Frontini y Escosteguy 2011; Lanzelotti y Bonaparte 2009; Politis et al. 2014; Politis y Madrid 1988; entre otros). La frecuente intervención de vizcachas y armadillos podría estar vinculada a que estos mamíferos ocupan lugares relativamente altos y cercanos al agua en este paisaje, localizaciones que también fueron seleccionadas por cazadores-recolectores en el pasado (Rafuse et al. 2017; Salemme 1987).

La morfología, tamaño y distribución de las cuevas registradas en el perfil estratigráfico y planta de excavación sugieren que la vizcacha fue el agente que tuvo mayor responsabilidad en las alteraciones postdepositacionales del sitio y del enterratorio humano en particular. Esta hipótesis también se ve apoyada por los numerosos huesos de este roedor en el sitio sin evidencia de aprovechamiento antrópico (Bellinzoni 2018). Los estudios actualísticos que hemos realizado con vizcachas (Bellinzoni 2018; Bellinzoni y Kaufmann 2014; Rafuse et al. 2017) muestran, al igual que en el sitio LM2; contextos con matrices sedimentarias mezcladas, agrupamientos de toscas, alta diversidad de pequeños vertebrados y frecuencia considerable de huesos de vizcachas (ver Bellinzoni 2018). Si bien es difícil establecer una cronología precisa de estas alteraciones, la posición estratigráfica del enterratorio 
en relación con la distribución de las cuevas y la presencia de materiales modernos en algunas de ellas, sugiere que la vizcachera se habría establecido en el sitio luego de los 2500 años AP y más probablemente hacia momentos actuales.

El análisis tafonómico y espacial del enterratorio permitió comprender la historia tafonómica compleja que presenta el sitio. Los estudios sobre la estructura espacial de los huesos humanos indican que se trata de un enterratorio primario, en el cual, el individuo fue depositado en posición decúbito ventral con los miembros inferiores flexionados. Si bien la posición de los enterratorios no ha sido un tema sistemáticamente abordado en los estudios bioarqueológicos de la región pampeana, el tipo de posición inferida no sería frecuente en la misma. Este sepultamiento habría implicado un pozo excavado a expensas de los sedimentos eólicos del borde de la laguna hacia ca. 2500 años C14 AP. Estos sedimentos contenían ya material arqueológico como lo evidencia el fechado de ca. 4900 años C14 AP en el perfil 1. Luego la historia del sitio no es muy clara, pero en forma reciente se estabiliza el sector comenzando a desarrollarse un perfil de suelo. Asimismo, el sitio se ve afectado por una intensa actividad de vizcachas que mezclaron sedimentos y horizontes de suelo. La acción de estos roedores habría desplazado pies, manos, columna vertebral, costillas y miembros delanteros del esqueleto. El desplazamiento de elementos óseos por fuera del área excavada sería la causa principal de la falta de completitud que presenta el esqueleto. Este desplazamiento, además, habría provocado la fractura de los elementos más frágiles del esqueleto como costillas, vértebras y escápulas. No obstante, el impacto de los mamíferos fosoriales en la preservación e integridad de las unidades esqueletarias habría sido bajo.

Otros enterratorios en el área de estudio

Con anterioridad a los trabajos de investigación realizados desde el año 2010 por este equipo de investigación, no se conocía información arqueológica en relación con la presencia de restos humanos en la cuenca del arroyo Salado. A partir de las investigaciones efectuadas en los últimos años se pudo determinar la presencia de al menos seis individuos. Dos de ellos fueron excavados sistemáticamente con metodologías arqueológicas, uno correspondiente al sitio arqueológico Laguna Seca (Kaufmann y González 2013) y el otro, el analizado en el presente trabajo. Por otra parte, la policía científica recuperó restos humanos en el sitio arqueológico LM1 que fueron analizados por profesionales del área de la bioantropología (Méndez 2011; Wiggenhauser 2014). Finalmente, en inmediaciones de la ciudad de Gral. Lamadrid, movimientos de suelo generados por obras de urbanización expusieron restos humanos pertenecientes a dos individuos que fueron recuperados por la policía científica y posteriormente reenterrados por la comunidad mapuche - tehuelche Peñi Mapu de Olavarría (Diario El Popular, 30 de septiembre de 2015). Recientemente, durante tareas de campo en el sitio Hangar (Barros et al. 2018) se recuperaron restos 
aislados en superficie que fueron re expuestos por acción de animales cavadores como peludo (Chaetophractus villosus) y mulita (Dasypus hybridus).

Los restos recuperados en el sitio LM1 corresponden a un masculino adulto joven (18 a 20 años) y a un individuo femenino adulto medio (40 a 49 años) (Wiggenhauser 2014). El fechado realizado sobre el individuo masculino arrojó una edad de ca. 400 años AP. En cuanto a los dos individuos re enterrados, no pudieron obtenerse datos de ningún tipo. El entierro primario de Laguna Seca fue asignado a un único individuo adulto de sexo femenino datado en ca. 580 años AP (Kaufmann y González 2013). Por otra parte, el entierro de LM2 constituiría una inhumación primaria con un fechado de ca. 2500 años ${ }^{14} \mathrm{C}$ $\mathrm{AP}$ que muestra una posición del esqueleto novedosa para la región pampeana. Este entierro nos informa no sólo acerca de las prácticas mortuorias sino también del estado de salud, ya que el individuo presentaba algunas patologías óseas de tipo degenerativo como eburnaciones y osteofitos.

Un aspecto importante es que los cráneos recuperados en LM1 y LM2 evidencian deformaciones que se ajustan a la cronología de los patrones observados en sitios del sur de la región pampeana (Barrientos y Perez 2002; Perez 2006; Berón y Luna 2009). La variante temprana de deformación craneal artificial del tipo fronto-occipital (también referida como tabular-oblicua, tabular erecta pseudocircular o tabular erecta planofrontal) aparece en enterratorios con cronologías del Holoceno medio y tardío inicial. Los sitios que han sido datados radiocarbónicamente y que presentan esqueletos con este tipo de deformación craneal son: Arroyo Seco 2, La Colorada, Laguna de Púan, Laguna Tres Reyes 1, La Toma, Tapera Moreira y La Lomita (ver Figura 1). Todos presentan cronologías que van entre los ca. 7000 y 2000 años C14 AP (Barrientos 2001; Berón y Baffi 2003; Madrid y Barrientos 2000; Perez 2006; Perez et al. 2009; Politis 1984; Politis y Madrid 2001). El otro tipo de deformación, referida como tabular erecta planolámbdica, en la cual existe una compresión en la zona del lambda y expansión del frontal, sería característica del Holoceno tardío final (Barrientos 1997; Madrid y Barrientos 2000; Perez 2006; Perez et al. 2009). Los sitios del sur de la región pampeana en los cuales se han recuperado individuos con este tipo de deformación y que presentan dataciones radiocarbónicas son: Médano La Enriqueta, Paso Mayor 2, Laguna Los Chilenos 1, Paso Alsina, La Petrona y Chenque 1. Las cronologías de estos sitios van entre los ca. 1000 y 300 años C14 AP (Barrientos 1997, 2001; Bayón et al. 2010; Berón y Baffi 2003; Carrera Aizpitarte et al. 2013; Martínez 2004; Martínez et al. 2007; Perez 2006; Scabuzzo 2010).

Finalmente, es necesario remarcar la importancia que poseen los estudios de procesos de formación de sitio para aportar información positiva y útil en la interpretación de los contextos arqueológicos. A partir de este trabajo pudieron comenzar a delinearse algunas tendencias en las prácticas mortuorias de las poblaciones que habitaron en lomadas próximas al arroyo Salado y a orillas de pequeñas lagunas de la cuenca. 


\section{Bibliografía citada}

Barrientos, G.

1997 Nutrición y dieta de las poblaciones aborígenes prehispánicas del sudeste de la Región Pampeana. Tesis Doctoral inédita. Facultad de Ciencias Naturales y Museo, Universidad Nacional de La Plata, La Plata, Buenos Aires.

2001 Una aproximación bioarqueológica al estudio del poblamiento prehistórico tardío del Sudeste de la Región Pampeana. Intersecciones en Antropología 2: 3-18.

Barrientos, G. y S. I. Perez

2002 La dinámica del poblamiento humano del Sudeste de la Región Pampeana durante el Holoceno. Intersecciones en Antropología 3: 41-54.

Barros, M. P., J. Bellinzoni y C. A. Kaufmann

2018 Análisis de los Instrumentos Líticos de los Sitios Laguna La Redonda y Laguna Muscar 2 (Partido de Gral. Lamadrid, Provincia de Buenos Aires). Intersecciones en Antropología 19(2): 111-122.

Bayón, C., G. Martínez, G. Armentano y C. Scabuzzo

2004 Arqueología del Valle inferior del Río Colorado. Sitio La Primavera. Intersecciones en Antropología 5: 39-55.

Bayón, C., R. Pupio, R., Frontini, R., Vecchi, y C. Scabuzzo

2010 Localidad arqueológica Paso Mayor: nuevos estudios 40 años después. Intersecciones en Antropología 11: 115-128.

Behrensmeyer, A. K.

1978 Taphonomic and ecological information from bone weathering. Paleobiology 4 (2): 150162.

Bellinzoni, J.

2018 Análisis zooarqueológico y tafonómico del sitio Laguna Muscar 2 (Pdo. De General Lamadrid, Pcia de Buenos Aires). Tesis de Licenciatura inédita. Facultad de Ciencias Sociales, Olavarría, Buenos Aires.

Bellinzoni, J. y C. A. Kaufmann

2014. Las vizcachas (Lagostomus maximus) como agentes tafonómicos. Libro de resúmenes del Séptimo Congreso de Arqueología de la Región Pampeana Argentina. p96. Ciudad de Rosario. 
Bernal, V., P. González, S. Perez y H. Pucciarelli

2008 Entierros humanos del noreste de Patagonia:Nuevos fechados radiocarbónicos. Magallania 36(2): 125-134.

Berón M. A. y E. I. Baffi

2003 Procesos de cambio cultural en los cazadores-recolectores de la provincia de La Pampa, Argentina. Intersecciones en Antropología 4: 29-43.

Berón, M. y L. Luna

2007 Modalidad de entierro en el sitio Chenque 1. Diversidad y complejidad de los patrones mortuorios de los cazadores recolectores pampeanos. En Arqueología en las Pampas (ed. por C. Bayón, N. Flegenheimer, M. I. González y M. Frère), pp. 129-141. Sociedad Argentina de Antropología, Bahía Blanca.

2009 Distribución espacial y cronológica de la deformación craneana tabular erecta en Pampa y Norpatagonia. En Arqueología de Patagonia: una mirada desde el último confín (ed. por M. Salemme, F. Santiago, M. Álvarez, E. Piana, M. Vázquez y E. Mansur), pp. 561-575. Editorial Utopías, Ushuaia.

Binford, L. R.

1981 Bones: Ancient Men and Modern Myths. Academic Press, New York.

Borella. F., Mariano, C. y C. Favier Dubois

2007 Procesos tafonómicos en restos humanos en superficie en la localidad arqueológica de Bajo de la Quinta, Golfo San Matías (Río Negro). En Arqueología de Fuego-Patagonia. Levantando piedras, desenterrando huesos... y develando arcanos (ed. por F. Morello, M. Martinic, A. Prieto y G. Bahamonde), pp. 403-410. Ediciones CEQUA, Punta Arenas.

Brain, C. K.

1981 The Hunters or the Hunted?: An Introduction to African Cave Taphonomy. University of Chicago Press, Chicago.

Buikstra, J. E. y D. H. Ubelaker

1994 Standards for Data Collection from Human Skeletal Remains. Arkansas Archaeological Survey Research Series No 44, Arkansas.

Carrera Aizpitarte, M., L. Luna, C. Aranda y M. Berón 2013 Médano La Enriqueta: un lugar de entierro de cazadores tardíos sobre el río Colorado (Dpto. Caleu Caleu, provincia de La Pampa). Revista del Museo de la Plata, Sección Antropología, 13(87): 167-184. 
Duday, $\mathrm{H}$.

2009 The Archaeology of the Dead. Lectures in Archaeothanatology. Oxbow Books, Southampton.

El Popular

(30 de septiembre de 2015). Restitución histórica de dos cuerpos aborígenes a comunidades originarias. Recuperado de http://www. elpopular.com.ar/eimpresa/222511/es-un-gesto-juridico-y-social-muy-importante-paratoda-la-sociedad.

Fidalgo, F., De Francesco, F. y U. Colado 1973 Geología superficial de las hojas Castelli, J. M. Cobo y Monasterio (Pcia. de Bs. As.) Actas V Congreso Geológico Argentino 4: 27-39, Córdoba.

Frontini, R. y C. Deschamps

2007 La actividad de Chaetophractus villosus en sitios arqueológicos. El Guanaco como caso de estudio. En Arqueología en las Pampas (ed. por C. Bayón; A. Pupio; M. I. González; N. Flegenheimer y N. M. Frère), pp. 439-451. Sociedad Argentina de Antropología, Buenos Aires.

Frontini, R. y P, Escosteguy

2011 Chaetophractus villosus: a disturbing agent for archaeological contexts. International Journal of Osteoarchaeology. Special Taphonomy Issue 22: 603-615.

González, M.

2013 Procesos de formación y efectos tafonómicos en entierros humanos: el caso del sitio Paso Alsina 1 en Patagonia Nororiental. Magallania 40 (1): 133-154.

2014 Procesos de formación y efectos tafonómicos en entierros humanos: el caso del sitio Arroyo Seco 2 en la región Pampeana, Argentina. Relaciones de la Sociedad Argentina de Antropología XXXIX (1): 175-202.

Gutiérrez, M. A.

2004 Análisis tafonómicos en el Área Interserrana (Provincia de Buenos Aires). Tesis Doctoral inédita. Facultad de Ciencias Naturales y Museo, Universidad Nacional de La Plata, La Plata, Buenos Aires.

Henderson, J.

1987 Factors determining the state of preservation of human remains. Death, Decay and Reconstruction. En Approaches to Archaeology and Forensic Science (ed. por A. Boddington, A. N. Garland y R. C. Janaway), pp. 43-54. Manchester University Press, Manchester. 
Johnson, E.

1985 Current developments in bone technology. En Advances in Archaeological Method and Theory (ed. por M. B. Schiffer), Vol. 8, pp.157-235. Academic Press, New York.

Kaufmann, C. A. y M. González

2013 Rescate arqueológico de restos óseos humanos en el sitio Laguna Seca 1 (Pdo. de Gral. Lamadrid, Pcia. de Buenos Aires). Revista del Museo de la Plata, Sección Antropología, 13(87): 125-136.

Klein, R. G. y K. Cruz-Uribe

1984 The Analysis of Animal Bones from Archaeological Sites. University of Chicago Press, Chicago.

Lanzelotti, S. L. y J. F. Bonaparte

2009 Contexto geoestratigráfico y procesos de formación del registro arqueológico en Cañada Honda: apuntes para su discusión y abordaje. En IV Congreso Argentino de Cuaternario y Geomorfología, XII Congresso da Associação Brasileira de Estudos do Quaternário, II Reunión sobre el Cuaternario de América del Sur (ed. por E. E. Fucks, C. Deschamps, C. G. Silva y E. J. Schnack), pp. 247-258. La Plata.

Lyman, R.L.

1994 Vertebrate Taphonomy. Cambridge University Press, Cambridge.

Lyman, R. L. y G. L. Fox

1989 A critical evaluation of bone weathering as an indication of bone assemblage formation. Journal of Archaeological Science 16: 293-317.

Madrid, P. y G. Barrientos

2000 La estructura del registro arqueológico del sitio Laguna Tres Reyes 1 (Provincia de Buenos Aires): nuevos datos para la interpretación del poblamiento humano del Sudeste de la Región Pampeana a inicios del Holoceno tardío. Relaciones de la Sociedad Argentina de Antropología XXV: 179-206.

Marín Arroyo, A. B.

2004 Análisis arqueozoológico, tafonómico y de la distribución espacial de la fauna de mamíferos de la Cueva de la Fragua (Santoña, Cantabria). Ediciones TGD, Santander.

Martínez, G.

2004 Resultados preliminares de las investigaciones arqueológicas realizadas en el curso inferior del río Colorado (partidos de Villarino y Patagones, provincia de Buenos Aires). En Aproximaciones contemporáneas a la arqueología pampeana. Perspectivas teóricas, 
metodológicas, analíticas y casos de estudio (ed. por G. Martínez, M. Gutiérrez, R. Curtoni, R., M. Berón y P. Madrid), pp. 275-292. Facultad de Ciencias Sociales (UNCPBA), Olavarría.

Martínez, G., G. Flensborg, P. Bayala y R. López

2007 Análisis de la composición anatómica, sexo y edad de dos entierros secundarios del sitio Paso Alsina 1 (Pdo. de Patagones, Pcia. de Buenos Aires). En Arqueología en las Pampas (ed. por C. Bayón, I. González, N. Flegenheimer, A. Pupio y M. Frére), pp. 41-58. Sociedad Argentina de Antropología, Buenos Aires.

Méndez, M. G.

2011 Informe Policía Científica. Ministerio de Justicia y Seguridad. Provincia de Buenos Aires. Ms. en archivo, Departamento de Arqueología, Facultad de Ciencias Sociales (UNCPBA), Olavarría.

Perez, S. I.

2006 El Poblamiento Holocénico del Sudeste de la Región Pampeana: Un Estudio de Morfometría Geométrica Craneofacial. Tesis Doctoral inédita. Facultad de Ciencias Naturales y Museo, Universidad Nacional de La Plata, La Plata, Buenos Aires.

Perez, S. I., C. Della Negra, P. Novellino, P. N. Gonzalez, V. Bernal, E. Cuneo y S. Hajduk 2009 Deformaciones artificiales del cráneo en cazadores-recolectores del Holoceno mediotardío del noroeste de Patagonia. Magallania: 37(2):77-90.

Politis, G. G.

1984 Arqueología del área Interserrana Bonaerense. Tesis Doctoral inédita. Facultad de Ciencias Naturales y Museo, Universidad Nacional de La Plata, La Plata, Buenos Aires.

Politis, G., Barrientos, G. y C. Scabuzzo

2014 Los entierros humanos de Arroyo Seco2. Estado actual de las investigaciones en el sitio arqueológico Arroyo Seco 2 (Partido de Tres Arroyos, provincia de Buenos Aires, Argentina). En Serie Monográfica del INCUAPA Nro. 6. (ed. por G. Politis, M. A. Gutiérrez y C. Scabuzzo), pp. 329-369. Facultad de Ciencias Sociales (UNCPBA), Olavarría.

Politis, G., y P. Madrid

1988 Un hueso duro de roer: análisis preliminar de la tafonomía del sitio Laguna Tres Reyes 1(Pdo. de Adolfo Gonzáles Chaves, Pcia. de Buenos Aires). En De Procesos, Contextos y Otros Huesos (ed. por N. Ratto y A. Haber), pp. 29-44. Facultad de Filosofía y Letras (UBA), BuenosAires.

2001 Arqueología Pampeana: Estado actual y perspectivas. En Historia Argentina Prehispánica, editado por E. E. Berberián y A. E. Nielsen. Vol. II: 737-814. 
Rafuse, D., C. A. Kaufmann, M. Gutiérrez, M. González, N. Scheifler, M.C. Álvarez y A. Massigoge

2017 Taphonomy of modern communal burrow systems of the Plains vizcacha (Lagostomus maximus, Chinchillidae) in the Pampas region of Argentina: implications for the fossil record. Historical Biology: 1-18.

Salemme, M.

1987 Paleoetnozoología del sector bonaerense de la región Pampeana, con especial atención a los mamíferos. Tesis doctoral inédita. Facultad de Ciencias Naturales y Museo, Universidad Nacional de La Plata, La Plata, Buenos Aires.

Scabuzzo C.

2010 Actividades, patologías y nutrición de los cazadores recolectores pampeanos. Tesis Doctoral inédita. Facultad de Ciencias Naturales y Museo, Universidad Nacional de La Plata, La Plata, Buenos Aires.

\section{Schiffer, M.}

1987 Formation processes of the archaeological record. University of New Mexico Press, Albuquerque.

Shipman, P.

1981 Life history of a fossil: an introduction to taphonomy and paleoecology. Harvard University Press, Cambridge.

Steele, G. y C. Bramblett

2005 The Anatomy and Biology of the Human Skeleton. Texas A\&M University Press, College Station.

Stodder, A. L. W.

2008 Taphonomy and the nature of archaeological assemblages. En Biological Anthropology of the Human Skeleton (ed. por M. A. Katzenberg y S. R. Saunders), pp. 71-114. Wiley-Liss Inc., New York.

Tani, M.

1995 Beyond the identification of formation processes: behavioral inference based on traces left by cultural formation processes. Journal of archaeology method and theory 2(3):231252. 
Todd, L. C.

1987 Taphonomy of The Horner II bone bed. En The Horner Site: The type site of the cody cultural complex (ed. por G. C. Frison y L. C. Todd, L. C), pp. 107-198. FL: Academy Press, Orlando.

Todd, L. y D. Stanford

1992 Application of conjoined bone data to site structural studies. En Piecing together the past: applications of refitting studies in archaeology (ed. por J. Hofman y J. Enloe), pp.21-35. BAR International Series 578, Oxford.

Todd, L. y G. Frison

1992 Reassembly of Bison skeleton from to Horner Site: A Study of Anatomical Refitting. En Piecing together the Past: Applications of refitting Studies in Archaeology (ed. por J. Hofman y J. Enloe), pp. 63-82. BAR International Series 578, Oxford.

Ubelaker, D. H.

1978 Human skeletal remains. Excavation, analysis, interpretation. Aldine Publishing Company, Chicago.

Villa, P. y E. Mahieu

1991 Breakage patterns of human long bones. Journal of Human Evolution 21: 27-48.

Walthall, J.

1999 Mortuary behavior and early Holocene land use in the North American midcontinent. North American Archaeologist 20(1):1-30.

White, T. y P. Folkens

2000. Human Osteology. Academic Press, San Diego.

Wiggenhauser, N.

2014 Aproximaciones a la Interpretación del Registro Bioarqueológico de Laguna Muscard, Provincia de Buenos Aires. Entre Pasados y Presentes IV. Estudios Contemporáneos en Ciencias Antropológicas. 